\title{
Fuzzy Swinging-up with Sliding Mode Control for Third Order Cart-Inverted Pendulum System
}

\author{
Belal A. Elsayed ${ }^{*}$, Mohsen A. Hassan, and Saad Mekhilef
}

\begin{abstract}
Cart Inverted Pendulum (CIP) system is a benchmark problem in nonlinear automatic control. In this paper, two third-order differential equations were derived to create a combining model for the cart-pendulum with its DC motor dynamics. Motor voltage was considered the system input in the presented model. The friction between the cart and rail was included in the system equations through a nonlinear friction model. Fuzzy Swinging-up controller was designed to swing the pendulum to the upright position, once reaching the upward position; Sliding Mode Controller (SMC) is activated, to balance the system. In order to verify the performance of the proposed SMC, a Linear Quadratic Regulator Controller (LQRC) was suggested and compared with the proposed SMC. Simulation and experimental results have shown a significant improvement of the proposed SMC over LQRC where, the pendulum angle oscillations were decreased by $80 \%$ in the real implementation.
\end{abstract}

Keywords: Fuzzy control, nonlinear systems, sliding mode control, third-order cart-inverted pendulum system.

\section{INTRODUCTION}

Sliding mode control is considered as a robust nonlinear control technique which has been applied widely for many applications $[1,2]$. The main advantages of this method are stability guarantee, external disturbance rejection and insensitivity to system parameters variation [3].

Cart-Inverted Pendulum (CIP) system is considered as one of the most significant benchmark problems in dynamics. As a nonlinear unstable system, it affords many challenging tasks to control engineers. Many practical restrictions exist with regard to controlling the system, including friction, cart rail limitation and control signal saturation. These limitations motivate researchers to study the system extensively.

Swinging-up and stabilization of the inverted pendulum is a common control problem. The main study on swing-up pendulum systems was conducted by Astrom

Manuscript received January 12, 2014; revised April 18, 2014; accepted May 13, 2014. Recommended by Associate Editor Sung Jin Yoo under the direction of Editor Ju Hyun Park.

The authors acknowledge the financial support by University of Malaya research grant (UMRG), under the grants number RG118/11AET and RP006E/13ICT.

Belal A. Elsayed is with the College of Engineering, AMA International University, 712 Bahrain (e-mail: baelsayed@amaiu. edu.bh).

Mohsen A. Hassan is with the Department of Engineering Design and Manufacturing, University of Malaya, Kuala Lumpur 50603, Malaysia and the Department of Mechanical Engineering Department, Assiut University, Assiut 71516, Egypt (e-mail mohsenegypt@um.edu.my).

Saad Mekhilef is with the Department of Electrical Engineering, University of Malaya, Kuala Lumpur 50603, Malaysia (email: saad@um.edu.my).

* Corresponding author. and Furuta [4]. Energy control method was adopted to control pendulum energy and swing the pendulum upward. However, cart rail limit was not considered in the described model. Chatterjee et al. [5] introduced a swinging-up controller based on the energy well principle to swing the pendulum within a restricted cart length. However, in the stabilization phase, only local stability was guaranteed due to the model approximation. Muskiniji and Tovorkin [6] proposed a fuzzy swinging up controller with a linear stabilization controller. The fuzzy swinging-up scheme exhibited better performance comparing with energy control strategy. However, the stability of the stabilization controller was not discussed. Tao et al. [7] proposed a fuzzy swing-up controller with parallel distributed pole assignment stabilization controller. Despite the validity of this controller, the DC motor dynamics was not included and the experimental investigation is still needed.

Several controllers were also proposed based on Sliding Mode Control to stabilize CIP. In [8], Chang and Hui proposed a decoupled fuzzy-sliding mode controller for CIP. In this approach, the whole system is decoupled into two subsystems (the cart and the pendulum); each subsystem has its own control target. Decoupling function is designed to ensure that the control signal will control both of subsystems. However, the proposed controller did not consider experimental limitations such as DC motor dynamics, friction, and cart length restriction. Lin and Mon [9] proposed a hierarchical fuzzy sliding mode controller for CIP system. Simulation results showed the effectiveness of their controller. Neural network decoupling controller for nonlinear systems was also introduced by Hung and Chung [10]. However, the decoupling techniques utilized in [9] and [10] were more complex compared with that utilized by Chang [8]. In addition the 
experimental verification is still needed. Tao et al. [11] proposed a fuzzy swinging-up controller with a sliding mode controller. Simulation results verified the effectiveness of this controller. More advanced controller based on time varying sliding surface controller was proposed by Yorgancioglu and Komurcugil [12]. Results showed improvement of the pendulum angle response in terms of speed convergence. However, the applied techniques in $[8,11,12]$ described CIP with two second-order equations. This procedure means that system input was the force applied on the cart. Such representation cannot be directly applied in an experimental environment because the actual system is driven by a DC motor.

In the present paper, a fuzzy swinging-up with sliding mode stabilization controller has been proposed to swing up and stabilize CIP. The fuzzy swing-up controller has been designed to swing up the system to the upward position within the cart limits. Then, when the pendulum reaches the upper position, Sliding Mode stabilization controller is applied. The sliding surface has been designed based on a third order representation for CIP.

After considering the DC motor dynamics, the overall system model has been derived. Two third order differential equations have been obtained to represent the system. The system input simply becomes the applied voltage on the DC motor, which means that the cart model has been combined with the DC motor in one mathematical representation. For switching between controllers, one move switch is designed to switch between the swinging-up and stabilization states. The proposed SMC controller has been compared with LQR technique, which was applied in [5] and [6], in order to show the effectiveness for SMC especially in balancing the system under nonlinear friction effects. Simulation and experimental results have been discussed to show the effectiveness of this controller especially in rejecting the friction effects and system parameters uncertainties.

The rest of paper is organized as follows. The system model is derived in Section 2. The fuzzy swing-up controller and sliding mode balancing controller are described in Section 3. The simulation results are presented in Section 4. Real-time implementation and experimental results are discussed in Section 5. The comparison of LQRC and SMC is performed and discussed in Section 6 . Finally, the conclusion is provided in Section 7.

\section{MATHEMATICAL MODEL}

\subsection{Pendulum model}

CIP system has two degrees of freedom, $X$ is the Cart displacement, and $\theta$ is the pendulum angle position, as shown in Fig. 1. If the cart mass and the pendulum mass were donated by $M$ and $m$, respectively. $L$ is the length between the pivot and the pendulum center of gravity C.G, $g$ is the acceleration of gravity, lis the pendulum mass moment of inertia with respect to its C.G., $F_{f}$ is the friction force between the cart and the rail and $q$ is the friction coefficient in the pendulum pivot. Based on D'Alembert's principle, the equations of motion are deduced to be:

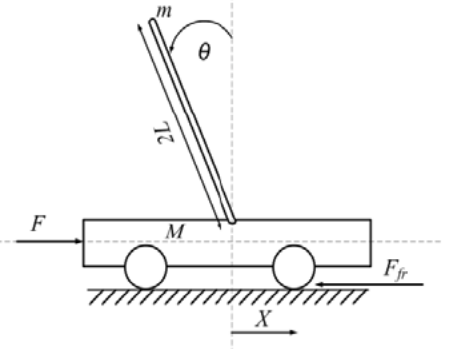

Fig. 1. Cart-pendulum system.

$$
\begin{aligned}
& F=(M+m) \ddot{X}+F_{f r}-m\left(L \ddot{\theta} \cos \theta-L \dot{\theta}^{2} \sin \theta\right), \\
& \left(I+m L^{2}\right) \ddot{\theta}=m g L \sin \theta+m L \ddot{X} \cos \theta-q \dot{\theta} .
\end{aligned}
$$

For the friction Force $F_{f r}$, most of the former work, dealing with the CIP, either has applied a viscous friction model (linear) or has neglected its effects. However, the friction phenomena encloses many terms such as Stribeck effects, static, Coulomb and viscous frictions [13]. Thus, exponential friction model is chosen, to address all listed friction parts, as follows:

$$
F_{f r}=\left\{\begin{array}{lrl}
\dot{X} \frac{F_{S}}{\dot{X}_{d}}, & \text { if } & |\dot{X}| \leq\left|\dot{X}_{d}\right| \\
F_{C}+\left(F_{S}-F_{C}\right) \exp \left(-\left|\frac{\dot{X}}{V_{S}}\right|\right) \operatorname{sgn}(\dot{X})+b \dot{X}, & \text { if } & |\dot{X}|>\left|\dot{X}_{d}\right|,
\end{array}\right.
$$

where $F_{S}$ is static friction force, $F_{C}$ is Coulumb friction force, $\dot{X}_{d}$ is the dead zone velocities, $V_{S}$ is Stribeck velocity, $n$ is form factor, and $b$ is the viscous friction coefficient.

\subsection{De motor model}

For the motor Circuit, if $V_{a}$ is the armature applied voltage (control voltage), $V_{e m f}$ is the back EMF voltage, and $R_{a}, L_{a}$ and $i$ are the armature resistance, inductance and current, respectively. $\omega$ is the angular velocity of the DC Motor, $T_{e}$ is the motor electromagnetic torque, $T_{J}$ is motor inertia torque, $T_{B}$ is damping torque and $T_{L}$ is motor load torque. The motor equations are

$$
\begin{aligned}
& V_{a}-V_{e m f}=i R_{a}+L_{a} \frac{d i}{d t}, \\
& V_{e m f=} K_{e} \omega, \\
& i=\frac{T_{e}}{K_{t}},
\end{aligned}
$$

$K_{t}$ is the motor torque constant. The relation between the cart linear velocity and the motor angular velocity is given by (7)

$$
\omega=\frac{\dot{X}}{r},
$$


where $r$ is the motor pulley diameter. The electromagnetic torque equation is

$$
T_{e}=T_{J}+T_{B}+T_{L},
$$

where

$$
\begin{aligned}
& T_{J}=J \dot{\omega}=J \frac{\ddot{X}}{r}, \\
& T_{B}=B \omega=B \frac{\dot{X}}{r}, \\
& T_{L}=F r .
\end{aligned}
$$

$J$ is the motor rotor mass moment of inertia, $B$ Motor rotor damping coefficient.

\subsection{Overall system model}

Here, two third order differential equations will be derived to describe the overall system, where the motor applied voltage $V_{a}$ is the system input. By substituting from (9), (10) and (11) in (8). And from (8) in (6) we get the current equation

$$
\begin{aligned}
i=\frac{T_{e}}{K_{t}}= & \frac{J \frac{\ddot{X}}{r}+B \frac{\dot{X}}{r}+[(M+m) \ddot{X}}{K_{t}} \\
& \frac{\left.+F_{f r}-m\left(L \ddot{\theta} \cos \theta-L \dot{\theta}^{2} \sin \theta\right)\right] r}{K_{t}} .
\end{aligned}
$$

Taking the time derivative of the last equation, equation (13) is obtained

$$
\begin{aligned}
\frac{d i}{d t} & =\frac{\left[(M+m) r+\frac{J}{r}\right] \dddot{X}+\frac{B}{r} \ddot{X}-m L r \ddot{\theta} \cos \theta}{K_{t}} \\
& \frac{+m L r \ddot{\theta} \dot{\theta} \sin \theta+2 m L r \ddot{\theta} \dot{\theta} \sin \theta+m L r \dot{\theta}^{3} \cos \theta+\dot{F}_{f r}}{K_{t}} .
\end{aligned}
$$

By substituting from (5), (12) and (13) in (4), we obtain

$$
\begin{aligned}
V_{a}= & {\left[\left[(M+m) r+\frac{J}{r}\right] \frac{L_{a}}{K_{t}}\right] \dddot{X} } \\
& +\left[\left[(M+m) r+\frac{J}{r}\right] \frac{R_{a}}{K_{t}}+\frac{B L_{a}}{r K_{t}}\right] \ddot{X} \\
& -\left[\frac{B R_{a}}{r K_{t}}+\frac{K_{e}}{r}\right] \dot{X}-\frac{r m L R_{a}}{K_{t}} \ddot{\theta} \cos \theta \\
& +\frac{r m L R_{a}}{K_{t}} \dot{\theta}^{2} \sin \theta-\frac{r m L L_{a}}{K_{t}} \dddot{\theta} \cos \theta \\
& +\frac{3 r m L L_{a}}{K_{t}} \ddot{\theta} \sin \theta+\frac{r m L L_{a}}{K_{t}} \dot{\theta}^{3} \cos \theta \\
& +\frac{R_{a}}{K_{t}} F_{f r}+\frac{L_{a}}{K_{t}} \dot{F}_{f r} .
\end{aligned}
$$

Equation (14) is considered as the main overall equation, describing the system states with the applied voltage on DC motor as an input.
From (2) we obtain

$$
\begin{aligned}
& \ddot{X}=-g \tan \theta+\frac{\left(I+m L^{2}\right)}{m L \cos \theta} \ddot{\theta}+\frac{q}{m L \cos \theta} \dot{\theta} \\
& \ddot{\theta}=\frac{m g L}{\left(I+m L^{2}\right)} \sin \theta+\frac{m L}{\left(I+m L^{2}\right)} \cos \theta \ddot{X}-\frac{q}{\left(I+m L^{2}\right)} \dot{\theta} .
\end{aligned}
$$

Taking the first derivative of (2), we get

$$
\begin{aligned}
\dddot{X}= & -g \dot{\theta}+\ddot{X} \tan \theta \dot{\theta}+\frac{q}{m L \cos \theta} \ddot{\theta}+\frac{\left(I+m L^{2}\right)}{m L \cos \theta} \dddot{\theta}, \\
\dddot{\theta}= & \frac{m g L}{\left(I+m L^{2}\right)} \cos \theta \dot{\theta}+\frac{m L}{\left(I+m L^{2}\right)} \dddot{X} \cos \theta \\
& -\frac{m L}{\left(I+m L^{2}\right)} \ddot{X} \sin \theta \dot{\theta}-\frac{q}{\left(I+m L^{2}\right)} \ddot{\theta} .
\end{aligned}
$$

Substituting from (15) and (17) in (14), we get the pendulum angle third order differential equation

$$
\begin{aligned}
\dddot{\theta}= & \frac{f_{3} \dot{\theta}-f_{4} \tan ^{2} \theta \dot{\theta}+f_{5} \frac{\tan \theta \dot{\theta} \ddot{\theta}}{\cos \theta}+f_{6} \frac{\tan \theta \dot{\theta}^{2}}{\cos \theta}}{\left[f_{1} \cos \theta-\frac{f_{2}}{\cos \theta}\right]} \\
& \frac{+f_{7} \frac{\ddot{\theta}}{\cos \theta}-f_{8} \tan \theta+f_{9} \frac{\dot{\theta}}{\cos \theta}+f_{10} \dot{X}-f_{11} \ddot{\theta} \cos \theta}{\left[f_{1} \cos \theta-\frac{f_{2}}{\cos \theta}\right]} \\
& +f_{12} \dot{\theta}^{2} \sin \theta+f_{13} \dot{\theta} \ddot{\theta} \sin \theta+f_{14} \dot{\theta}^{3} \cos \theta \\
& \left.+f_{15} F_{f r}+f_{16} \cos \theta-\frac{f_{2}}{\cos \theta}\right] \\
& {\left[f_{1} \cos \theta-\frac{1}{\cos \theta}-\frac{f_{2}}{\left[f_{1} \cos \theta-\frac{f_{2}}{\cos \theta}\right]} V_{a} .\right.}
\end{aligned}
$$

The constants values $f_{1 \rightarrow 16}$ are listed in Appendix A. Equation (19) is then rewritten

$$
\begin{aligned}
\dddot{\theta}= & \alpha_{1}(\ddot{\theta}, \dot{\theta}, \theta, \dot{X})+\beta_{1}(\theta) V_{a}, \\
\alpha_{1}= & \frac{f_{3} \dot{\theta}-f_{4} \tan ^{2} \theta \dot{\theta}+f_{5} \frac{\tan \theta \dot{\theta} \ddot{\theta}}{\cos \theta}+f_{6} \frac{\tan \theta \dot{\theta}^{2}}{\cos \theta}}{\left[f_{1} \cos \theta-\frac{f_{2}}{\cos \theta}\right]} \\
& +f_{7} \frac{\ddot{\theta}}{\cos \theta}-f_{8} \tan \theta+f_{9} \frac{\dot{\theta}}{\cos \theta}+f_{10} \dot{X}-f_{11} \ddot{\theta} \cos \theta \\
& +\left[f_{1} \cos \theta-\frac{f_{2}}{\cos \theta}\right] \\
& +f_{12} \dot{\theta}^{2} \sin \theta+f_{13} \dot{\theta} \ddot{\theta} \sin \theta+f_{14} \dot{\theta}^{3} \cos \theta \\
& {\left[f_{1}+f_{16} \dot{F}_{f r}\right.} \\
& {\left[f_{1} \cos \theta-\frac{f_{2}}{\cos \theta}\right] }
\end{aligned}
$$




$$
\beta_{1}=-\frac{1}{\left[f_{1} \cos \theta-\frac{f_{2}}{\cos \theta}\right]},
$$

we substitute from (16) and (18) in (14) to obtain the cart position third-order differential equation.

$$
\begin{aligned}
\dddot{X}= & \frac{-\bar{f}_{3} \cos ^{2} \theta \dot{\theta}+\bar{f}_{4} \ddot{X} \sin \theta \cos \theta \dot{\theta}+\bar{f}_{5} \cos \theta \sin \theta}{\bar{f}_{2} \cos ^{2} \theta-\bar{f}_{1}} \\
& +\frac{\bar{f}_{6} \cos ^{2} \theta \ddot{X}+\bar{f}_{7} \cos \theta \dot{\theta}+\bar{f}_{8} \ddot{X}+\bar{f}_{9} \dot{X}+\bar{f}_{10} \dot{\theta}^{2} \sin \theta}{\bar{f}_{2} \cos ^{2} \theta-\bar{f}_{1}} \\
& \frac{+\bar{f}_{11} \dot{\theta} \sin ^{2} \theta+\bar{f}_{12} \dot{\theta} \sin \theta \cos \theta \ddot{X}+\bar{f}_{13} \dot{\theta}^{3} \cos \theta+\bar{f}_{14} F_{f r}}{\bar{f}_{2} \cos ^{2} \theta-\bar{f}_{1}} \\
& \frac{+\bar{f}_{15} \dot{F}_{f r}}{\bar{f}_{2} \cos ^{2} \theta-\bar{f}_{1}}-\frac{1}{\bar{f}_{2} \cos ^{2} \theta-\bar{f}_{1}} V_{a} .
\end{aligned}
$$

The constants values $\bar{f}_{1 \rightarrow 15}$ are listed in Appendix A. Equation (23) is rewritten in as

$$
\begin{aligned}
& \dddot{X}=\alpha_{2}(\ddot{X}, \dot{X}, \dot{\theta}, \theta)+\beta_{2}(\theta), \\
& \dddot{X}=\frac{-\bar{f}_{3} \cos ^{2} \theta \dot{\theta}+\bar{f}_{4} \ddot{X} \sin \theta \cos \theta \dot{\theta}+\bar{f}_{5} \cos \theta \sin \theta}{\bar{f}_{2} \cos ^{2} \theta-\bar{f}_{1}} \\
& \frac{+\bar{f}_{6} \cos ^{2} \theta \ddot{X}+\bar{f}_{7} \cos \theta \dot{\theta}+\bar{f}_{8} \ddot{X}+\bar{f}_{9} \dot{X}+\bar{f}_{10} \dot{\theta}^{2} \sin \theta}{\bar{f}_{2} \cos ^{2} \theta-\bar{f}_{1}} \\
& \frac{+\bar{f}_{11} \dot{\theta} \sin ^{2} \theta+\bar{f}_{12} \dot{\theta} \sin \theta \cos \theta \ddot{X}+\bar{f}_{13} \dot{\theta}^{3} \cos \theta+\bar{f}_{14} F_{f r}}{\bar{f}_{2} \cos ^{2} \theta-\bar{f}_{1}} \\
& \frac{+\bar{f}_{15} \dot{F}_{f r}}{\bar{f}_{2} \cos ^{2} \theta-\bar{f}_{1}} \\
& \beta_{2}=-\frac{1}{\bar{f}_{2} \cos ^{2} \theta-\bar{f}_{1}} \text {. }
\end{aligned}
$$

\section{CONTROLLER DESIGN}

The fuzzy swing-up controller is discussed in the first subsection of this current section. The design of stabilization SMC is discussed in the succeeding subsection.

\subsection{Fuzzy swing-up controller}

The main idea of swinging-up depends on the energy control [4].The pendulum energy equals the sum of the kinetic and potential pendulum energies. In this system the pendulum energy is

$$
E=I_{P} \dot{\theta}^{2}+m g L \cos \theta,
$$

where $I_{P}$ is the pendulum mass moment of inertia around the pivot point. According to (27), the pendulum energy depends on the pendulum angle and the pendulum angular velocity. By increasing the pendulum energy the pendulum could be swung up to the upright position. Fuzzy logic controller is used to swing the pendulum up. In this fuzzy controller the pendulum is aimed to swing up to the upright position by increasing its energy. The cart rail and the control action limitations should be considered. Three input variables are chosen. The pendulum angle $\theta$, pendulum angular velocity $\dot{\theta}$ and the displacement $X$, are the fuzzy controller inputs. The DC motor control voltage $V_{a(\text { swing-up })}$ is the output variable.

The membership functions of the input and output variables are shown in Figs. 2 to 5. Fig. 2 presents the five membership functions selected for the angle. The rectangular membership function (1) represents the pendulum angle $\theta$, where $(\pi / 2 \leq \theta<3 \pi / 2)$; accurate measurement is not necessary at this state. The pendulum angular velocity $\dot{\theta}$, in Fig. 3 is represented by two membership functions, namely, $\mathrm{N}$ (clockwise) and $\mathrm{P}$ (counterclockwise). The cart displacement $X$ is represented by two triangular and one trapezoidal membership function as shown in

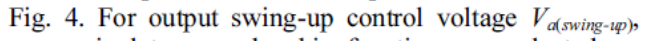
seven singleton membership functions are selected, as shown in Fig. 5, to represent the applied control voltage on the DC motor. The positions of the singleton membership functions are selected to minimize swing-up time.

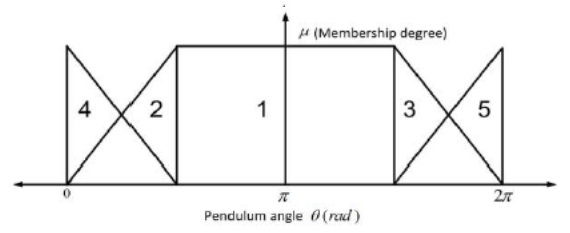

Fig. 2. Pendulum angle membership functions.

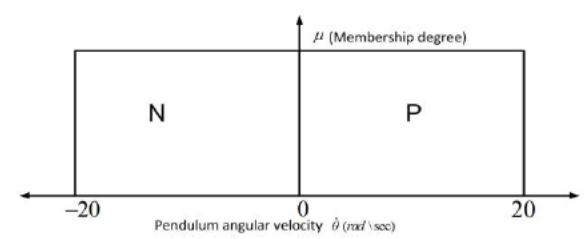

Fig. 3. Pendulum angular velocity membership functions.

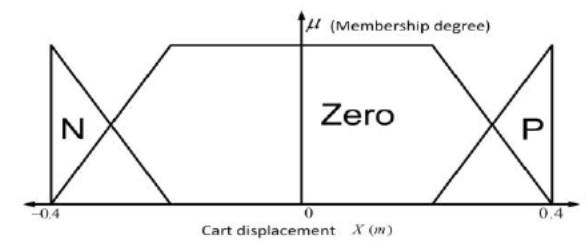

Fig. 4. Cart position membership functions.

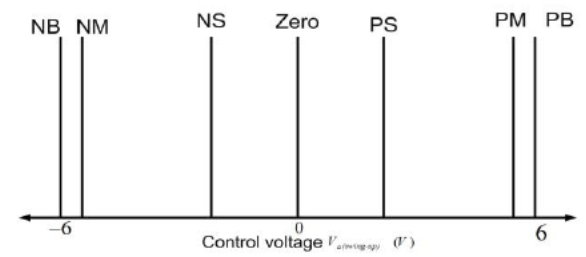

Fig. 5. Output control voltage membership functions. 
The swing-up controller is designed based on 30 fuzzy rules. The rules consequents are chosen to increase the pendulum energy to reach the upward position energy. During the swinging-up, the cart rail limitation should be considered. Each three rules are designed at the same Pendulum angle $\theta$ and angular velocity $\dot{\theta}$, with consideration of the cart position.

For instance, if the pendulum angle is 1 and the pendulum angular velocity is $\mathrm{N}$, the three rules are developed as follows: First, without consideration of the cart limits, the logical swing-up control action should be PB. Then, the cart position membership functions (N,P and Zero) will be considered to form the three rules, for each rule $\theta$ and $\dot{\theta}$ are constant ( 1 and $\mathrm{N}$, respectively).

Rule 1: If $\theta$ is 1 and $\dot{\theta}$ is $\mathrm{N}$ and $X$ is $\mathrm{P}$, then $V_{a(\text { swing- } \imath p)}$ is Zero.

This rule means that the pendulum is located in the downward half cycle $(\pi / 2 \leq \theta<3 \pi / 2)$ and rotates clockwise. As mentioned above, the logical swing-up control decision should be PB. However, as the cart is located at the positive side of the rail $(X$ is $P)$, the rule consequent should be $\boldsymbol{V} \boldsymbol{a}_{\text {(swing-up) }}=\mathbf{0}$ to keep the cart within the limits.

Rule 2: If $\theta$ is 1 and $\dot{\theta}$ is $\mathrm{N}$ and $X$ is Zero, then $V_{a(\text { swing-up })}$ is PM.

The cart is located in the middle of the rail ( $X$ is zero). Thus, the control action should be to move the cart in the positive direction with a medium force. The rule consequent should be $\boldsymbol{V} \boldsymbol{a}_{(\text {swing-up) }}$ is PM.

Rule 3: If $\theta$ is 1 and $\dot{\theta}$ is $\mathrm{N}$ and $X$ is $\mathrm{N}$, then $V_{\text {a(swing- }}$ vp) is PB.

The rule consequent should be $\boldsymbol{V} \boldsymbol{a}_{(\text {swing-up) }}$ is PB because the cart is located at the negative side of the rail $(\boldsymbol{X}$ is $N$ ).

The other 27 rules are selected through the same procedure. The controller allows the pendulum to reach the upward position while the cart remains within the restricted limits.

\subsection{Sliding mode stabilization controller}

Sliding Mode Controller is designed based on the third order derived mode. From the system model in (20) and (24), and if $D_{I}$ and $D_{2}$ are bounded external disturbances, the entire system model will have the following form

$$
\begin{aligned}
& \dddot{\theta}=\alpha_{1}+\beta_{1} V_{a}+D_{1}, \\
& \dddot{X}=\alpha_{2}+\beta_{2} V_{a}+D_{2},
\end{aligned}
$$

where $\alpha_{1}, \alpha_{2}, \beta_{2}$ and $\beta_{1}$ are nonlinear functions of the system states. The control law is designed based on the sliding surface. The general equation of the sliding surface $S$ is [14]

$$
S(x, t)=\left(\frac{d}{d t}+C\right)^{n-1} \cdot x,
$$

where $x$ is the system sate, $n$ is the system order and $C$ is a constant value. The sliding surface will be constructed based on the third order derived model where $n=3$. In this case (CIP), two sliding surfaces, $S_{I}$ for the pendulum subsystem and $S_{2}$ for the Cart subsystem, are considered. where

$$
\begin{aligned}
& S_{1}=C_{1}^{2} \theta+2 C_{1} \dot{\theta}+\ddot{\theta}, \\
& S_{2}=C_{2}{ }^{2} X+2 C_{2} \dot{X}+\ddot{X},
\end{aligned}
$$

$C_{1}$ and $C_{2}$ are positive constants. Sliding surfaces $S_{1}$ and $S_{2}$ are constructed based on the constants $C_{1}$ and $C_{2}$. Appropriate selection of these constants values will achieve the desired response.

The control law is designed based on the sliding surfaces. Since only one control action is available, the Pendulum angle will be considered as primary control target and the cart position is the secondary target. Initially, the controller is designed to achieve the primary target where $S_{l}=0$. An intermediate function is utilized to link between the secondary and primary targets. This function will achieve the cart subsystem stability if the pendulum stability is reached.

The control is designed based on Lyapunov like function $V$

$$
V=\frac{1}{2} S_{1}^{2} .
$$

As it is known from sliding mode theorem, in order to achieve the system stability the control law must match the following reaching condition

$$
\dot{V}=\dot{S}_{1} S_{1} \leq-\eta\left|S_{1}\right|,
$$

where $\eta>0$, this condition ensures that the system will be driven into the sliding mode. The control law will be derived as follow, from (34)

$$
\dot{S}_{1} \cdot \operatorname{sgn}\left(S_{1}\right) \leq-\eta .
$$

From (31)

$$
\dot{S}_{1}=C_{1}^{2} \dot{\theta}+2 C_{1} \ddot{\theta}+\ddot{\theta} .
$$

Substituting with (36) in (35)

$$
\begin{aligned}
& \left(C_{1}^{2} \dot{\theta}+2 C_{1} \ddot{\theta}+\alpha_{1}+\beta_{1} V_{a}+D_{1}\right) \cdot \operatorname{sgn}\left(S_{1}\right) \leq-\eta, \\
& \left(C_{1}^{2} \dot{\theta}+2 C_{1} \ddot{\theta}+\alpha_{1}+D_{1}\right) \cdot \operatorname{sgn}\left(S_{1}\right)+\beta_{1} V_{a} \cdot \operatorname{sgn}\left(S_{1}\right) \leq-\eta,
\end{aligned}
$$

$$
\begin{aligned}
& \left(\frac{C_{1}^{2} \dot{\theta}+2 C_{1} \ddot{\theta}+\alpha_{1}+D_{1}}{\beta_{1}}\right) \cdot \operatorname{sgn}\left(S_{1} \beta_{1}\right) \\
& +V_{a} \cdot \operatorname{sgn}\left(S_{1} \beta_{1}\right) \leq-\frac{\eta}{\left|\beta_{1}\right|} .
\end{aligned}
$$

From (39), the control law could be written in form

$$
V_{a(\text { stablize })}=\frac{-C_{1}^{2}-2 C_{1} \ddot{\theta}-\alpha_{1}}{\beta_{1}}-K \operatorname{sgn}\left(S_{1} \beta_{1}\right),
$$

where

$$
K \geq \frac{D_{1}+\eta}{\left|\beta_{1}\right|} .
$$

The first term of the control law is estimated from the 
system model, and it will be donated as $\hat{V}_{a(\text { stablize })}$, where:

$$
\hat{V}_{a(\text { stablize })}=\frac{-C_{1}^{2}-2 C_{1} \ddot{\theta}-\alpha_{1}}{\beta_{1}} .
$$

This form of the control signal guarantee the stability for the pendulum subsystem since the reaching condition is achieved and the sliding motion will occur. The control action $V_{a(\text { stabilize), }}$ as it is shown in (40), has a highfrequencies switching because of the $\mathrm{Sgn}$ function. To overcome this problem, a boundary layer will be formed by replacing $S g n$ function with Sat function as follows:

$$
V_{a(\text { stablize })}=\widehat{V}_{a(\text { stablize })}-K \operatorname{sat}\left(\frac{S_{1} \beta_{1}}{\phi}\right),
$$

where

$$
\operatorname{sat}\left(\frac{S_{1} \beta_{1}}{\phi}\right)= \begin{cases}\operatorname{sgn} \frac{S_{1} \beta_{1}}{\phi}, & \text { if }\left|\frac{S_{1} \beta_{1}}{\phi}\right| \geq 1 \\ \frac{S_{1} \beta_{1}}{\phi} & \text { if }\left|\frac{S_{1} \beta_{1}}{\phi}\right|<1,\end{cases}
$$

where $\Phi$ is upper limit of the boundary layer function.

This kind of control scheme will be capable of rejecting all the high-frequencies and solve the chattering problem.

The control law in (41) can only guarantee the pendulum angle stability. The control objective is to move the pendulum and the cart subsystems to the sliding surfaces $S_{1}$ and $S_{2}$, respectively, where the overall system stability could be achieved. In order to do that, an intermediate function $Z$ has been introduced to link between the two subsystems sliding surfaces $S_{1}$ and $S_{2}$ [7]. The function $Z$ design is introduced as follows:

First, the first sliding surface will be reformed to be

$$
S_{1}=C_{1}^{2}(\theta-Z)+2 C_{1} \dot{\theta}+\ddot{\theta},
$$

where $Z$ is a function of $\mathrm{S}_{2}$ which means that the sliding surface $S_{2}$ was incorporated into the sliding surface $S_{1}$ through $Z$ function. The new sliding surface has changed the control target from $\theta=0, \dot{\theta}=0$ and $\ddot{\theta}=0$ to $\theta=z$, $\dot{\theta}=0$ and $\ddot{\theta}=0$. The objective $\left(S_{2}=0\right)$ is now embedded in the main control target through the variable $Z$ which is defined

$$
Z=\operatorname{sat}\left(\frac{S_{2}}{\phi_{z}}\right) \cdot Z_{U},
$$

where $Z_{U}$ is the upper limit of the function; $\phi_{z}$ is the function boundary layer. $Z$ is abounded oscillatory function decays to zero. When $Z$ reach zero, $S_{1}$ will be zero according to (43).

3.3. Switching between swinging-up and stabilization control

A one-move switch is developed to switch between the swing-up and stabilization controllers. This switch ensures that the fuzzy swing-up controller is activated only one once. When the pendulum reaches the upward point, the stabilization controller is activated permanently. Switch output $\left(\mathrm{V}_{\mathrm{a}}\right)$ is represented as follows:

$$
V_{a}= \begin{cases}V_{a(\text { swing-up })}, & \text { if }(0<\theta<2 \pi), \text { and }(N<1) \\ V_{a(\text { stablize })}, & \text { if }(\theta \geq 2 \pi \text { or } \theta \leq 0), \text { or }(N \geq 1)\end{cases}
$$

Where $N$ is an integer counter that counts the numbers of the upward position at $(\theta=0)$.

\section{SIMULATION RESULTS}

Simulation was performed with MATLAB. Simulink was utilized to solve and simulate the CIP system dynamics provided by (20) and (24). With the fuzzy logic toolbox in Matlab Simulink, the fuzzy swing-up controller was applied to swing the pendulum upward. Two different stabilization control schemes, namely, SMC and LQRC, were compared. Both controllers (SMC and LQRC) were tested with the fuzzy controller in the swing-up phase. For testing purposes, nonlinear friction force between the cart and rail was considered according to (3). This force acts as an external disturbance on the controller. Cart rail limit was set to $\pm 0.4 \mathrm{~m}$, and motor saturation voltage was set to $\pm 6 \mathrm{~V}$. All CIP systems and friction force parameters are listed in Table. The CIP system and LQRC parameters were selected to keep the controller within the system limits. The selected controller parameters for SMC were chosen $C_{I}=5.5, C 2$ $=3.1, K=15, \Phi=8 \times 104, \Phi_{z}=19$, and $Z_{U}=0.98$.

In addition, and for comparing purposes, LQR controller are designed to stabilize CIP following the same algorithm that was applied in $[4,5]$. The selected parameters for LQRC were $R=\operatorname{diag}\left[\begin{array}{llll}400 & 1 & 2500 & 1\end{array}\right]$, $Q=4$, and generated feedback gain vector $K_{L}=[-10$ $-12.990 .517 .4]$.

The pendulum angle response for the fuzzy swing-up controller with SMC is shown in Fig. 6. Pendulum

Table 1. System parameters.

\begin{tabular}{|c|c|c|}
\hline Parameter & Value & Unit \\
\hline$M$ & 0.882 & $\mathrm{~kg}$ \\
\hline$m$ & 0.32 & $\mathrm{~kg}$ \\
\hline$L$ & 0.3302 & $\mathrm{~m}$ \\
\hline$I$ & $7.88 \times 10^{-8}$ & $\mathrm{~kg} \cdot \mathrm{m}^{2}$ \\
\hline$g$ & 9.8 & $\mathrm{~m} / \mathrm{s}^{2}$ \\
\hline$q$ & 0.0001 & $\mathrm{~N} . \mathrm{s} / \mathrm{rad}$ \\
\hline$L_{a}$ & $0.18 \times 10^{-3}$ & $\mathrm{H}$ \\
\hline$R_{a}$ & 2.6 & $\mathrm{Ohm}$ \\
\hline$J$ & $3.9 \times 10^{-7}$ & $\mathrm{~kg} \cdot \mathrm{m}^{2}$ \\
\hline$B$ & $8 \times 10^{-7}$ & $\mathrm{~N} . \mathrm{m} . \mathrm{s} / \mathrm{rad}$ \\
\hline$K_{t}$ & 0.00676 & $\mathrm{~N} . \mathrm{m} / \mathrm{A}$ \\
\hline$K_{e}$ & 0.00676 & $\mathrm{~V} . \mathrm{s} / \mathrm{rad}$ \\
\hline$r$ & $6.35 \times 10$ & $\mathrm{~m}$ \\
\hline$F_{s}$ & 0.1 & $\mathrm{~N}$ \\
\hline$F_{c}$ & 0.08 & $\mathrm{~N}$ \\
\hline$V_{s}$ & 0.1 & $\mathrm{~m} / \mathrm{s}$ \\
\hline$b$ & 1.3 & $\mathrm{~N} . \mathrm{s} / \mathrm{m}$ \\
\hline$n$ & 4 & - \\
\hline$\dot{X}_{d}$ & 0.05 & $\mathrm{~m} / \mathrm{s}$ \\
\hline & & \\
\hline
\end{tabular}




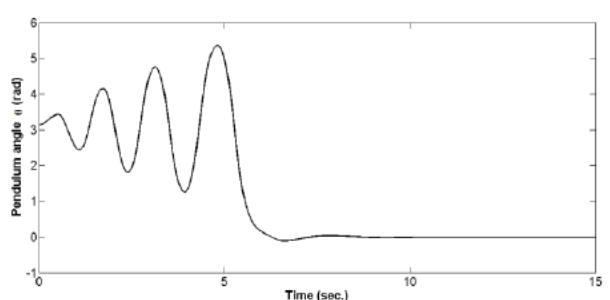

Fig. 6. Pendulum angular position response for fuzzy swing-up with SMC.

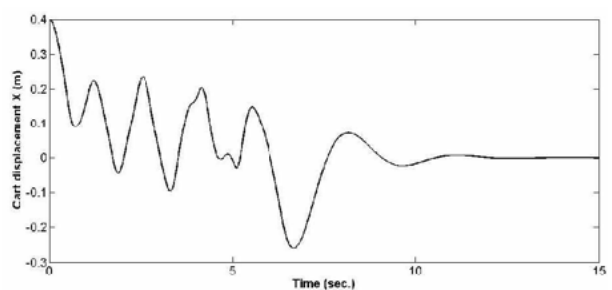

Fig. 7. Cart position response for fuzzy swing-up with SMC.

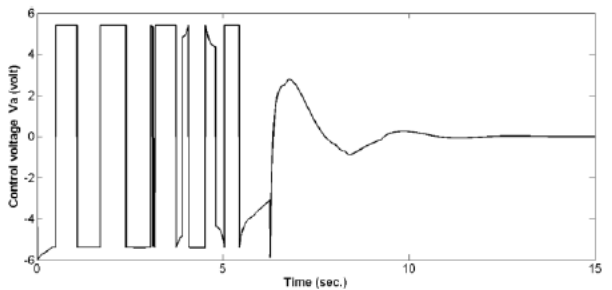

Fig. 8. Control voltage response for fuzzy swing-up with SMC.

swing-up time is within $6 \mathrm{~s}$. The figure shows the effectiveness of the SMC in stabilizing the system in spite of nonlinear friction forces. Cart displacement and control signal response are shown in Figs. 7 and 8, respectively. Stability is achieved when both curves decay into zero.

The results of the fuzzy swing-up controller with LQRC are shown in Figs. 9 to 11. However, the results reveal oscillations in cart position and control voltage responses. $L Q R C$ cannot achieve system stability within the simulation time because of friction uncertainties.

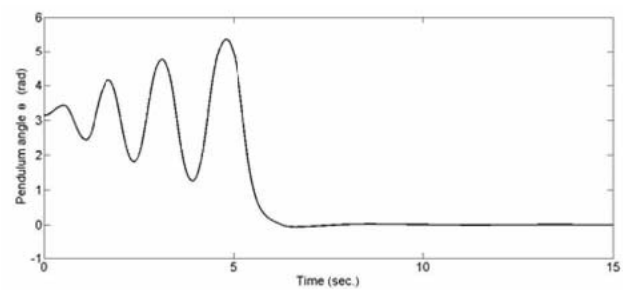

Fig. 9. Pendulum angular position response for fuzzy swing-up with LQRC.

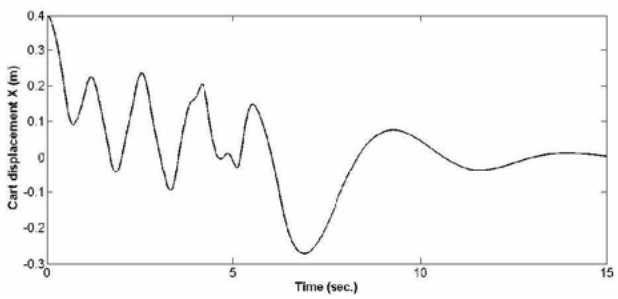

Fig. 10. Cart position response for fuzzy swing-up with LQRC.

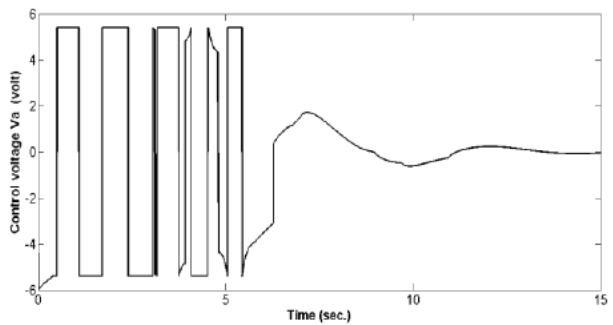

Fig. 11. Control voltage response for fuzzy swing-up with LQRC.

\section{EXPERIMENTAL RESULTS}

The experiment was conducted with inverted pendulum model system IP02 supplied by Quanser Limited. The electromechanical setup consisted of the cart-pendulum mechanical setup, DC motor, and two incremental encoders to measure the pendulum angle and cart position. The two encoders had a resolution of $0.0015 \mathrm{rad} /$ count and $2.275 \times 10^{-5} \mathrm{~m} /$ count, respectively. The controller setup included personal computers, an $\mathrm{AD} / \mathrm{DA}$ data acquisition card, and a Quanser power module (model UPM 800) to amplify the control signal. A least square fitting algorithm was utilized for velocity and acceleration estimation. Fitting was performed with a third-order fitting curve for eight counts (LSF 3/8) [17].

The control algorithms were established with Matlab Simulink and QuaRC real-time toolbox developed by Quanser with a clock frequency of $1 \mathrm{kHz}$. The fuzzy swing-up controller was tested experimentally with two stabilization controllers, SMC and LQRC. The selected controller parameters for SMC real-time implementation were $C_{1}=4, C_{2}=2, K=5, \Phi=2.2 \times 10^{3}, \Phi_{z}=4$, and $Z_{u}=0.9$. The selected controller parameters for LQRC were $R=\operatorname{diag}\left[\begin{array}{lllll}400 & 1 & 2500 & 1\end{array}\right], Q=1$, and generated feedback gain vector $K_{L}=\left[\begin{array}{llll}-20 & -21.6 & 124.96 & 23.2\end{array}\right]$.

Figs. 12 to 14 present the results of the actual implementation of the fuzzy swing-up controller with SMC. The pendulum was swung upward within $6 \mathrm{~s}$ before SMC was applied. The pendulum was balanced in the upward position; stability was noted. Cart displacement and control signal were driven near the equilibrium point with minimal oscillation.

The fuzzy swing-up controller was tested with LQRC. The results are shown in Fig. 17. The pendulum took $6 \mathrm{~s}$ 




Fig. 12. Experimental result for pendulum angular position with Fuzzy swing up and SMC.

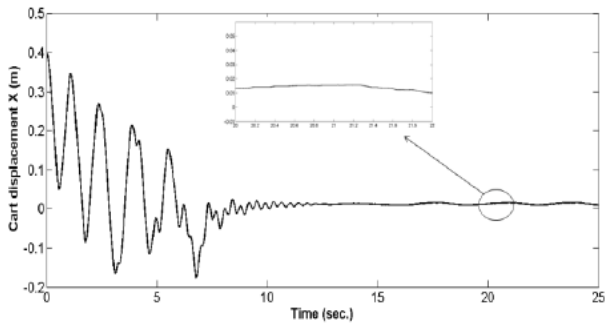

Fig. 13. Experimental result for cart position for Fuzzy swing up with SMC.

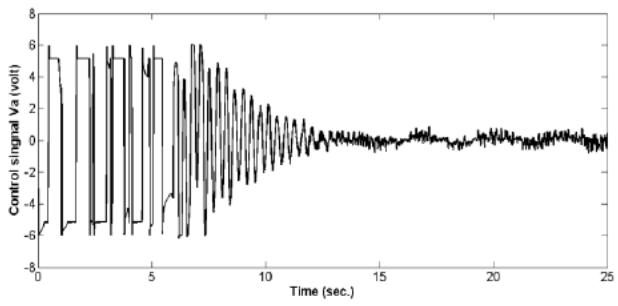

Fig. 14. Experimental result for control voltage for Fuzzy swing up with SMC.

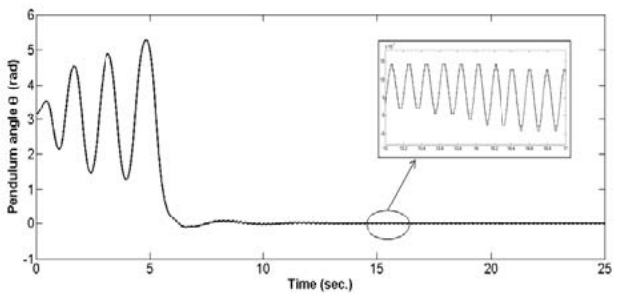

Fig. 15. Experimental result for pendulum angular position with Fuzzy swing up with LQRC.

to reach the upward position before $\mathrm{LQRC}$ was applied. Some oscillations were observed in the pendulum angle response because of the friction effect and other uncertainties. The cart response exhibits oscillations and steady-state errors. Fig. 17 shows the control signal for the LQRC where high overshoot values can be observed.

The steady state error could be in the cart position plots (Figs. 13 and 16) is due to many sources such as the

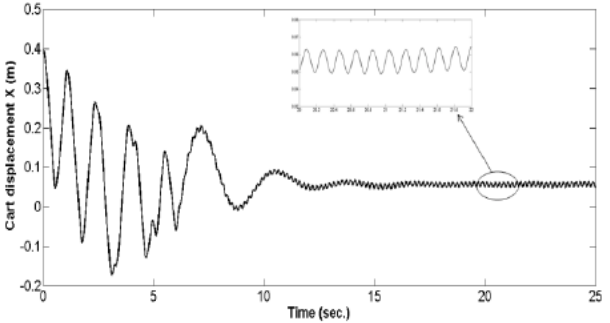

Fig. 16. Experimental result for cart position with Fuzzy swing up with LQRC.

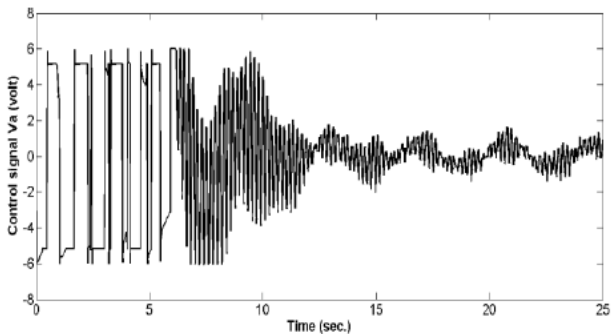

Fig. 17. Experimental result for control voltage for Fuzzy swing up with LQRC.

real nonlinear friction effect which is still has unknown terms depending on the temperature and the cart pinion and the rail materials. In addition the cart pinion has been shunted with the motor shaft, thus some relative motion is expected especially in the aggressive motion of the motor. Moreover, some errors caused by motor backlash, signal noise, digital quantization have not been considered in the simulation.

\section{COMPARISON RESULTS AND DISCUSSION}

Simulation and experimental comparison were performed with the SMC and LQR applied experimentally in related literature. The aim of the comparison is to show the validity and effectiveness of SMC compared with LQR, especially in terms of steady-state response. LQR is simpler in design compared with SMC and does not experience the chattering problem. Selecting between LQR (linear) and SMC (nonlinear) depends on the designer and the applications itself. SMC is preferred in robust and precise control applications, and LQR is suitable for simple controller structures. Disturbance rejection can also be utilized with $L Q R$ to improve controller response.

An external disturbance with a value of $0.1 \mathrm{rad}$ and duration of $1 \mathrm{~s}$ was applied after $20 \mathrm{~s}$ during the simulation. The pendulum and cart responses are shown in Figs. 18 and 19, respectively. SMC produced a faster pendulum angular response and exhibited better ability to reject disturbance compared with $\mathrm{LQRC}$. The maximum overshoot was reduced by $30 \%$ when SMC was applied. The cart response revealed the robustness of SMC

\section{Link to Full-Text Articles :}


http://umexpert.um.edu.my/file/publication/00005361_115165.pdf

http://works.bepress.com/saad mekhilef/27/ 